\title{
General practitioners often used emotional responses for "referrals away" and intellectual decision making for "referrals to" in patients with mild depression and/or anxiety
}

Nandy S, Chalmers-Watson C, Gantley M, et al. Referral for minor mental illness: a qualitative study. Br J Gen Pract 2001 Jun; $1: 461-5$.

QUESTION: What are the decision making processes of general practitioners (GPs) for referring patients with mild depression and/or anxiety?

Design

Qualitative study using grounded theory.

\section{Setting}

East London and Essex, UK.

\section{Participants}

23 GPs who were 30-61 years of age (mean age $44 \mathrm{y}$, $65 \%$ men) were selected using purposive sampling that maximised the variety of GPs with respect to age, sex, country of undergraduate training, employment status (principal, assistant, or locum), area of practice, and interest in mental health.

\section{Methods}

2 GPs interviewed participants by asking open ended questions about the interviewees' management of minor mental illness. Interviews were audiotaped and transcribed, and the data were analysed using a grounded theory approach to generate explanatory concepts and categories. The process was iterative; after 23 interviews, no new concepts were generated. The findings were presented to 12 GPs to help assess field validity.

\section{Main findings}

2 main categories were generated regarding the referral process. Conduits/containers were 2 distinct referral strategies. GPs who deemed that minor mental illness was part of their general practice load used the containment strategy to prevent what they considered an inappropriate burden on other agencies. GPs who used a conduit strategy saw themselves more in a diagnostic (followed by triage) role and felt that these patients were best managed by others. Many GPs used both strategies in their practice. Most GPs described an approach in which they would see the patients initially for a period and then sometimes refer. Reasons for referral included lack of progress and poor rapport with the patient, with accompanying feelings of frustration, irritation, or anger. Containment behaviour was enhanced by interest in minor mental illness and by confidence in dealing with particular mental health problems. Referrals to/ referrals away describe the 2 types of reasons for referral. "Referrals to" involved proactive reasons (eg, the particular skills of a counsellor or patient desire to be referred). These were often for specific situations (eg, drug addiction). "Referrals away" were often the result of the GP's emotions (eg, frustration, anger, and irritation). Other reasons for "referrals away" included lack of time, failure of GP management, and instances when the GP thought the patient would not want to discuss personal details with someone they knew well.

\section{Conclusions}

General practitioners who saw patients with mild depression and/or anxiety used a combination of containment (managing it in the general practice) or conduit (referring to a mental health professional) strategies. Emotional responses were prominent in decisions to "refer away," whereas "referring to" usually involved a more thoughtful, intellectual decision.
Source of funding: in part, Claire Wand

Fund of the British Medical Association.

For correspondence: Dr S Nandy,

Department of General Practice and Primary Care, St Bartholomew's and Royal London School of Medicine and Dentistry, Queen Mary and Westfield College, London E1 4 NS, UK. Fax $+44(0) 207882$ 6396.

\section{COMMENTARY}

The study by Nandy et al is represents the growing interest in using qualitative research to understand the underlying motivations for medical staff's decisions and occupational emotional hazards. These concerns are related to burnout, which Maslach defined as "the cost of caring." It is interesting to recall Balint's classical concept of the "drug effect" of medical intervention, ${ }^{2}$ which is similar to the unspecific factor of all psychotherapies that targets the patient's need for containment, attention, and being heard.

The"referral away"reaction is similar to the psychoanalytic concept of counter transference (ie, the reactions induced by the patient's mental illness in the doctor's mind or behaviour). Dr Breuer sent his famous patient Anna O to his colleague, Dr Sigmund Freud, because he was disturbed by her seductive attitudes. Freud did not"refer away"his patient but, instead, used his own reactions and emotions to better understand her problems. This is exactly what is proclaimed today as proper qualitative research: the observer as part of the field to be observed.

With respect to the role of GPs in minor mental illness, no universal agreement exists about whether GPs should receive training in appropriate systematic mental health intervention, span of action, and personal limits. Different clinical settings, healthcare programmes, and national policies have dealt with the problem in different ways. In some institutions, postgraduate formal training in family medicine assumes a holistic approach to health and illness. In other cases, institutional routine provides a "ventilation" room, in which weekly meetings are held to discuss emotional difficulties stemming from contact with patients. (Traditional "Balint groups" are an example of this.) Another solution is having an interdisciplinary team always available in health stations for consultations about minor mental health problems. In other settings, co-consultation (ie, that performed simultaneously by medical and mental health professionals) allows a better distribution of the "weight" of the patient's needs according to the training of each. Undergraduate students can be warned about the emotional hazards of the profession and given information on warning signals, healthy ways of performing one's tasks, guidelines for dealing with different kinds of patients, and training in communication skills. Finally, doctors can also be trained to pay attention to their own counter transference reactions and taught to recognise them as part of the clinical picture of a given complaint.

Denise Defey, $\mathrm{PhD}$ University of Uruguay Montevideo, Uruguay, South America

1 Maslach C. Burnout: the cost of caring. Englewood Cliffs, NJ: Prentice-Hall Press, 1982.

2 Balint M. A study of doctors: mutual selection and the evaluation of results in a training programme for family doctors. London: Lippincott, 1966. 